\title{
Investigation of small motors operating under the Huber effect
}

\author{
A. P. Lauterbach, W. L. Soong and D. Abbott \\ Department of Electrical and Electronic Engineering and \\ Centre for Biomedical Engineering (CBME) \\ University of Adelaide \\ Adelaide, SA 5005, Australia
}

\begin{abstract}
The Huber effect is an interesting and potential useful means for creating extremely small and simple motors. It is based on the observation that torque is produced when current is passed through a rotating ball bearing. This paper reviews the alternative explanations for its operation and describes the design, construction and characterization of two prototype ball-bearing motors based on high precision miniature ball bearings. A key limitation of earlier work has been difficulties in repeatability due to rapid wear of the motor. This was overcome by using a data acquisition system to record the dynamic acceleration characteristics and hence predict acceleration torque versus speed characteristics.
\end{abstract}

Keywords: Huber Effect, ball-bearing motor, micromotor.

\section{INTRODUCTION}

The event of MEMS technology has identified an almost unbounded wave of possibility in the fields of medicine, communication, molecular level material exploration, microrobotics, space exploration, defence, mechanics and so on. Understandably this arrival brings the challenge of developing compatible microfabrication processes, behavioural modeling and control methods, materials with the desired characteristics and high accuracy design techniques.

Large motors based on the Huber effect are well known to have poor performance characteristics due to the large currents required - however, it is likely that as the size of the motor is scaled down, the current accordingly scales. Therefore an open question is whether micro Huber motors might have useful properties. To date there has been little work in characterizing the motor to fully understand its operation. This paper describes the problems involved in testing such a motor and shows preliminary results of our ongoing work.

The Huber effect was named in honour of J. Huber ${ }^{1}$, who in 1959 discovered the presence of an additional rotational force on a pair of conducting wheels when supplied with electric current and driven along rails. The additional force was observed to accelerate the wheels regardless of whether alternating current (AC) or direct current (DC) was used. The ball-bearing motor was first described by Milroy ${ }^{2}$ (1967) on the basis of the Huber effect. It consisted of a metal shaft mounted with a ball bearing at each end as shown in Figure 1. When AC or DC is passed through the assembly and if the shaft is given an initial starting torque it will continue to spin in the direction in which it was started. The motor is capable of rotating freely in either direction.

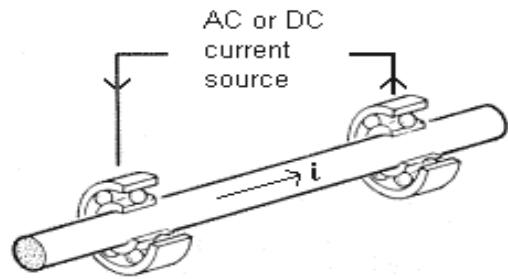

Figure 1. Ball-bearing motor. Current $I$ causes the shaft to revolve at speed $\omega$. After Ref. [3]. 
Despite its exceptional simplicity the ball-bearing motor has remained essentially unexplained and uncharacterized since its discovery. Several theories have been presented, but lack conclusive experimental evidence. Hence the aim of this paper is to investigate the physics behind the electromechanical device by accurately and quantitatively characterize its operation.

Although the ball-bearing motor exhibits poor torque and efficiency and is generally not self-starting, it has exciting possible applications in the field of microelectromechanical systems (MEMS). In this situation, its simplicity has advantages over most other more complex conventional motor designs, which would be more difficult to fabricate to the desired microscopic size.

\section{PREVIOUS HYPOTHESES}

Many have postulated about the physics behind the Huber effect, however no one paper or theory provides conclusive experimental evidence or disproves all other theories. Therefore the exact origin of the effect has remained a mystery. Three main hypotheses are: 1) an electromagnetic effect, 2) thermal expansion of the balls within the bearing cases and 3) spark discharge between the ball and race.

\subsection{The Electromagnetic Effect}

A paper by Gruenberg ${ }^{3}$ first attributed the motor phenomenon observed by Milroy to an electromagnetic effect. Consider a single ball in the bearing having volume current density $\mathrm{J}_{0}$ and magnetic field $\mathrm{B}_{0}$ due to the electric current flowing between the inner and outer race contacts. Assuming the ball has angular velocity $\omega$ then a secondary volume current density $\mathrm{J}_{1}$ will be induced because of the motion of the primary magnetic field $\mathrm{B}_{0}$. This causes a redistribution of charge and an associated magnetic field $B_{1}$ and electric field $E_{1}$. Although the interaction of these currents with the $B_{0}$ field will produce radial force, the major source of torque is a result of interactions between $\mathrm{J}_{0}$ and $\mathrm{B}_{1}$, and $\mathrm{J}_{1}$ and $\mathrm{B}_{0}$. Gruenberg showed that the torque was in the direction of the vector $\omega$ and therefore the motor develops rotational torque only once it is running and has no starting torque. Extending this theory using the corresponding static Maxwell equations revealed a torque equation directly proportional to both speed and the square of the current,

$$
T=k \cdot I^{2} \cdot \omega
$$

where $T$ is the torque produced, $k$ is a constant determined from physical properties of the ball, $\omega$ is the angular velocity and $I$ is the supply current. The speed and current relationship was then given as,

$$
I=\sqrt{a+b \cdot \omega}
$$

where $a$ and $b$ are constants.

These relationships were proven algebraically incorrect by Weenink ${ }^{4}$ and supported later by Moyssides and Hatzikonstantinou, who established that the first-order torque was in fact zero. Weenink also found that second-order torque calculations on the ball were again equal to zero. Moyssides and Hatzikonstantinou presented a slightly different explanation based on the electromagnetic forces resulting from the interactions of the applied current and induced currents and their magnetic fields. The magnetic field $\underline{B}_{0}$ and its induced current $\underline{\mathrm{J}}_{0}$ being caused by the flow of current in the motor shaft, balls and vicinity. Rigorous torque calculations involving a system of Maxwell's equations found the main contribution is due to the force,

$$
F=\underline{J} \times \underline{B}_{0}
$$

where $\underline{J}$ is the current density produced by the system whilst rotating and $\underline{B}_{0}$ is the magnetic field associated with primary current. They conclude in agreement with the square law equation (1) for the torque of the ball bearing motor and a non-linear speed-current relationship. 
Watson, Williams and Crimp ${ }^{7}$ disagreed with the above explanations as their experimental work showed a direct linear relationship between the developed torque and current. Their experiments were carried out with the motor immersed in kerosene to minimise arcing, pitting and corrosion and to help maintain a constant temperature environment.

\subsection{Thermal Expansion}

A completely different theory was presented by Marinov", who postulated that the ball bearing motor is "not an electromagnetic motor but a thermal engine." Heat generated at the point of contact between the ball and inner and outer ball races is due to increased ohmic resistance causing a physical dilation of the ball. When the motor is stationary the resultant elongation of the balls causes the bearing to seize, however with an initial spin it produces a torque in the direction of rotation, thus sustaining movement. This action happens as a continual process involving all balls in electrical contact with the bearing housing. Marinov also suggested that the occasional spontaneous starting of the motor was due to deviations in the race surface. Hence according to the thermal expansion theory self-starting would not be achievable with absolute smoothness and geometric perfection.

One of the most obvious flaws of this theory is that thermal effects generally have long time constants. This means that the rate of elongation and contraction of the balls to achieve even a moderate speed would seem impossible. Although the thermal expansion theory has been met with scepticism, its significance to isolating the true origin of the Huber effect should not be automatically disregarded without experimental evidence.

\subsection{Plasma Discharge}

Polivanov, Netushil and Tatarinova ${ }^{9}$ published a paper in 1973 attributing the Huber effect to spark discharge occurring between the lagging surface of the metal ball and race during rotation. The voltage causing the plasma stems from Lenz's law, stating "An induced EMF will tend to cause a current to flow in such a direction as to oppose the cause of the induced EMF." 10 The forces arise because of the interaction of induced currents within the magnetic field disturbing the symmetry of the current and flux distribution set up by past point contacts. The continual process is depicted schematically for the case of a wheel on guide conductor (rail) and axle in Figure 2.

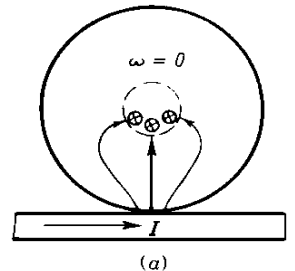

(a)

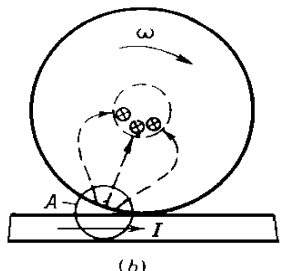

Figure 2. a) Spread of current in cross-section of a stationary wheel. b) New current distribution as wheel is rotated. After Ref.

$[1]$.

The nature of the forces produced is a combination of the reactive output of ions from the metal surface, the positive transfer of momentum by the same ions that are impacted in the spark breakdown, and thermal expansion of the gases behind the balls. In a more recent paper by Netushil ${ }^{11}$, an expression for the force of repulsion between the ball and inner race was derived and takes the form,

$$
F=k \cdot I^{2}
$$

where $k$ depends on the surface materials and the form of the welding arc. The paper did not determine the associated torque and current relations. 


\subsection{Conclusions from Our Previous Investigations}

Two different configurations of ball-bearing motor were experimented on by B. Thompson $e t$ al ${ }^{20}$ - namely, the external rotating cylinder motor and the internal rotating shaft motor. The main difference between the two topologies is that the external cylinder motor has a stationary inner bearing race and rotating outer race with attached cylinder. Whereas the internal shaft motor has a fixed outer ball race with rotating inner race and shaft. They found that the internal rotating shaft motor was the most practical design, enabling torque and speed measurements using a $9 \mathrm{~V}$-dc motor and optical shaft encoder.

They identified deionized water, kerosene and silicone oil as effective heat sink liquids. Rusting of the metal components was detected when running the ball-bearing motor in water and therefore, in this paper, tests are carried out using kerosene and silicone oil only.

To prove or disprove the plasma discharge theory, a ball-bearing motor was placed within a vacuum tube. Air was extracted from the tube and the motor set in motion using a magnetic clutch. The experiment was carried out in a darkroom so that the event of electrical arcing could be easily observed. However sparking was not witnessed, strongly suggesting that the effect is not a result of spark breakdown.

\section{BALL-BEARING MOTOR DESIGN}

Historically the ball-bearing motor has exhibited several limiting and quite destructive attributes. Extremely high currents (10$50 \mathrm{~A})$ are required to achieve motoring action even for a structure of small dimensions. Heat generation and effective thermal dissipation measures are therefore of major concern. Under extreme heat, the metal components of the motor expand and cause the seizure of the bearings. Electrical arcing also occurs between the balls and bearing races causing pitting of the ball surface, further adding to bearing degradation.

\subsection{Heat Sink Fluid}

To counteract or at least minimize the degrading effects of arcing that occurs between the balls and bearing housing and to reduce the effects of expansion of metal components, the motor has been designed to operate whilst immersed in a fluid. Kerosene and silicone oil (SF96-5) have been isolated as appropriate heat sink liquids because they exhibit low viscosity, low conductivity and high flash point, which are important properties for minimising liquid drag loss and spark discharge.

A fluid tank was formed from transparent perspex with slotted sides to facilitate quick insertion and removal of the various motors under investigation.

\subsection{Motor Design}

The first step in developing a small ball-bearing motor of the internal rotating shaft topology, as shown in Figure 1, was to identify an abundant supply of small diameter, high precision bearings with minimal friction and maximum number of balls. Computer hard-drive bearings proved to be the ideal source. Two bearings in the reader arm of each hard-drive allow the pivotal action that the heads require to be able to access tracks on the disk. Faulty hard-drives were collected from computer recyclers at zero cost and a variety of bearing sizes and types were obtained.

The prototype design incorporated part of the aluminium reading arm as a mounting in which the bearings were pressed as shown in Figure 3 (a). The rotor was machined from aluminium and has a $4 \mathrm{~mm}$ diameter extension above the top bearing to provide a means for attaching an optical encoder code wheel for rotor speed measurement. Preliminary motor tests were carried out on this motor revealing several critical issues. The motor would not sustain rotation and was even observed to oscillate around approximately $45^{\circ}$ suggesting misalignment in the shaft. The oscillation was particularly interesting because it indicated that sufficient torque was generated to overcome the friction and inertial torques during direction changes. The second issue was that the lightweight aluminium shaft was creating insufficient rotational momentum to allow continued rotor rotation. 
To improve shaft and bearing alignment a second design was constructed with features shown in Figure 3 (b). The top bearing mounting was removed and replaced with a cylindrical bakelite spacer to ensure both bearings were perpendicular to each other. The bakelite material was chosen for its high temperature tolerance and zero conductivity. A new rotor was fashioned from brass to increase the rotor weight and thus produce a flywheel effect whilst remaining rust resistant.

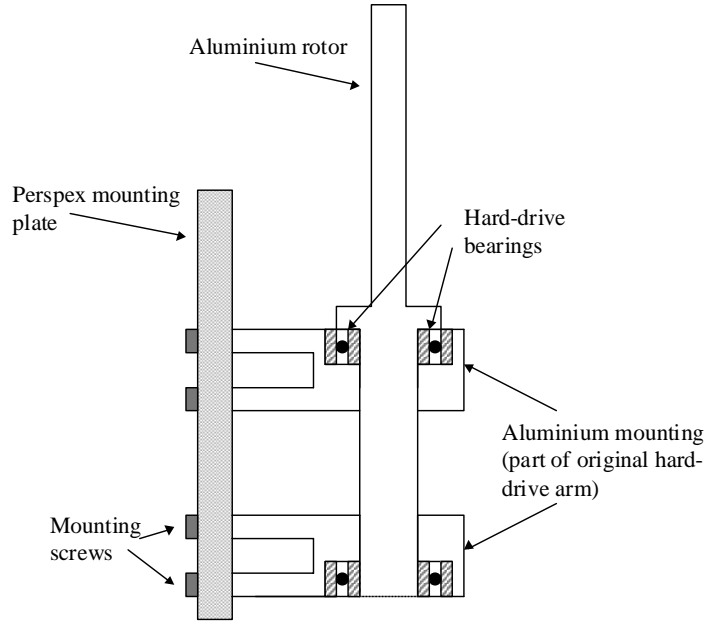

(a)

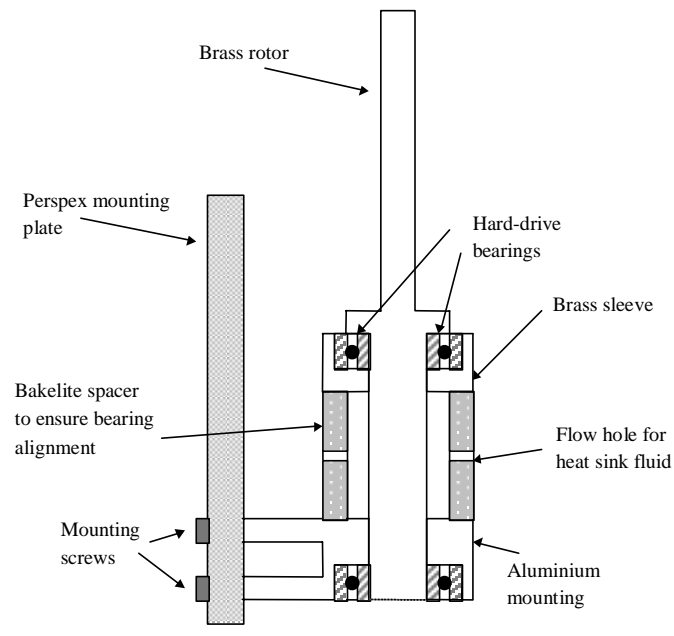

(b)

Figure 3. Ball-bearing motor designs. a) Initial design. b) Improved design.

Greater appreciation for the size and geometry of the ball-bearing motor can be gained from figure 4 below. Notice that electrical current is delivered to the motor via the copper braid and exits through a thin brass plate clamped between the bottom mounting and perspex bracket.

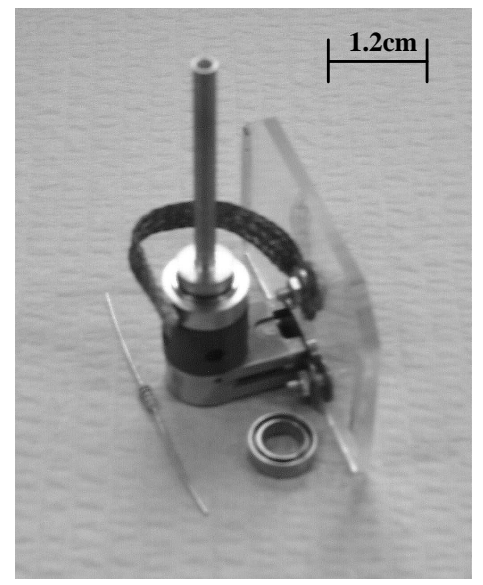

Figure 4. Photograph of second ball-bearing motor.

Included in the photograph is one of the hard-drive bearings and a resistor to show the size of the motor. 


\section{EXPERIMENTAL PROCEDURE AND METHODOLOGY}

This section describes the techniques and apparatus developed in the execution of practical experiments. However detailed explanation of the circuitry will not be presented.

\subsection{LabVIEW Data Acquisition}

LabVIEW (Laboratory Virtual Instrument Engineering Workbench) is a software package developed by National Instruments and was utilized to perform the data acquisition. LabVIEW uses a graphical language, $\mathrm{G}$, to create programs in block form. It includes libraries of functions and development tools designed specifically for data acquisition and instrument control.

Using LabVIEW enabled simultaneous sampling of various voltage and current waveforms to determine motor speed, torque and current relationships. Recorded values are output to a sequential data file, which can be readily imported into spreadsheet or data analysis software such as MATLAB. Real time waveforms are displayed in LabVIEW's interactive front panel, allowing observation of the values and possible trends instantaneously.

\subsection{Speed Measurement}

Speed and motor current measurement was realized using the apparatus depicted in figure 5. For currents less than 20A AC or DC a Hall Effect current transducer was used which converts the current values into voltage levels which can be measured and scaled appropriately using LabVIEW. For currents above 20A a shunt resistance was used and its value determined by the maximum deliverable supply current and the maximum accepted voltage on the data acquisition hardware.

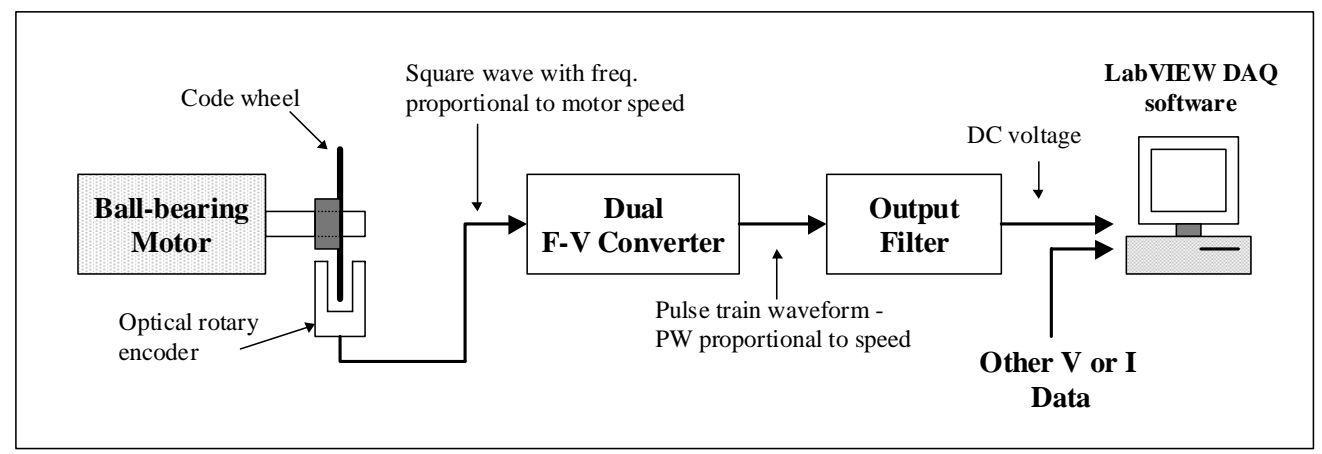

Figure 5. Block diagram of speed-current measurement system.

The optical rotary encoder uses the code wheel attached to that motor shaft to produce a square wave output signal with frequency directly proportional to the code wheel resolution (number of slots $N$ in outer rim) and the speed of the rotating motor shaft in revolutions per minute (rpm). The encoder output then feeds into the frequency-to-voltage converter and low pass filter to convert the square wave input signal to a DC voltage output whose amplitude is dependent on the input frequency and hence motor speed. Calibration of the device gives the scaling factors in $\mathrm{rpm} / \mathrm{V}$ used to convert voltage levels to speed values.

Figure 6 is the block diagram view of the LabVIEW VI (Virtual Instrument) implemented to record speed and current data. The DAQ object is a VI in itself and performs the continuous scanning of the specified analog input ports at a rate defined by the controllers on the front panel display. Data is stored in an array and accessed via indexing particular columns. Voltage level quantities are then split up, converted to speed and current values via appropriate scaling factors, plotted on the VI front panel and saved to a file in a spreadsheet format. The LabVIEW software can be edited easily to allow measurement of any quantity provided it can be converted into a voltage signal between $0-5 \mathrm{~V}$ for acquisition. 


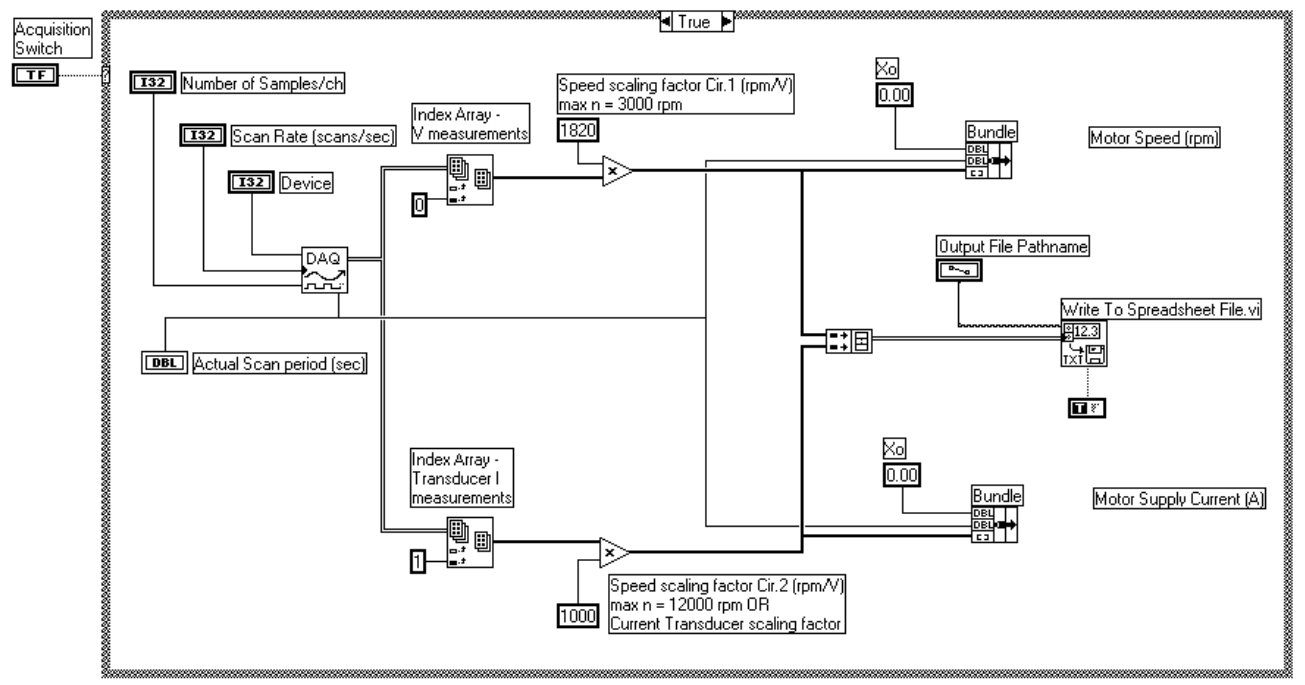

Figure 6. Block diagram representation of speed-current LabVIEW virtual instrument. Indicates the flow of data through voltage level acquisition, speed and current calculation, waveform display and saving to file.

\subsection{Torque Measurement}

Total system torque can be expressed as the sum of the friction and windage torques and the acceleration torque produced by the ball-bearing motor,

$$
T_{\text {Total }}=T_{\text {Friction\&Windage }}+T_{\text {Acceleration }}
$$

The acceleration torque was measured using the angular acceleration $\alpha$ of the ball-bearing motor and its moment of inertia $J$. A speed-time characteristic (from zero rpm to steady state speed) was generated using the LabVIEW speed measurement program, this was then differentiated to obtain acceleration using the relationship,

$$
\alpha=\frac{d \omega}{d t} \approx \frac{\Delta \omega}{\Delta t}
$$

where $\Delta \mathrm{t}$ is the actual scan period defined by the data acquisition and $\alpha$ is in $\mathrm{rad} / \mathrm{s}^{2}$. The moment of inertia of the system was then calculated taking only the rotor into account and assuming the bearing balls and inner race would have negligible effect. Acceleration torque could then be evaluated using the following expression,

$$
T_{\text {Acceleration }}=J \cdot \alpha
$$

The friction torque characteristic was obtained manually by using a small DC motor to drive the unexcited ball-bearing motor whilst in the kerosene bath. Therefore the friction torque comprises of bearing and liquid drag frictional losses. The DC motor armature current was measured for increasing values of speed which were converted to torque via eqn (8),

$$
T_{\text {Friction\&Windage }}=k \cdot I_{\text {arm }}
$$

where $k$ represents the back EMF constant for the DC motor determined through no-load and blocked-rotor tests. 
Corresponding points on both characteristics were summed to obtain the total torque produced by the ball-bearing motor as a function of angular velocity.

\section{EXPERIMENTAL RESULTS}

\subsection{Preliminary Speed Results (Prototype Ball-bearing Motor)}

Speed test trials were carried out on the prototype ball-bearing motor immersed in silicone oil. Although the tests were not successful in terms of useful speed data, they did alert to several serious problems; shaft misalignment as discussed in section 3.1, evidence of rusting on the aluminium rotor and large spark discharges observed within the fluid. At this point the conductivity of the silicone oil was questioned. Table 1 shows the results of conductivity tests performed.

The results reveal that the silicone oil does not have as high a volume resistance as expected or specified in the data sheet. For these reasons kerosene has been adopted as the only heat sink liquid to be used, having zero conductivity.

Table 1. Experimental conductivity measurement results.

\begin{tabular}{|c|c|c|c|}
\hline Heat sink fluid & Meter settings & Volume Resistance (M $\Omega$-cm) & Description \\
\hline \multirow[t]{3}{*}{ Silicone Oil } & $250 \mathrm{~V} / 100 \mathrm{M} \Omega$ & 0.4 & \multirow{3}{*}{$\begin{array}{l}\text { Silicone oil at ambient } \\
\text { temperature }\left(25^{\circ} \mathrm{C}\right)\end{array}$} \\
\hline & $500 \mathrm{~V} / 200 \mathrm{M} \Omega$ & 0.38 & \\
\hline & $1000 \mathrm{~V} / 400 \mathrm{M} \Omega$ & 0.46 & \\
\hline \multirow[t]{3}{*}{ Silicone Oil } & $250 \mathrm{~V} / 100 \mathrm{M} \Omega$ & 0.125 & \multirow{3}{*}{$\begin{array}{l}\text { Pre-heated silicone oil } \\
\left(80^{\circ} \mathrm{C}\right) \text { to evaporate } \\
\text { any absorbed } \mathrm{H}_{2} \mathrm{O} \text {. }\end{array}$} \\
\hline & $500 \mathrm{~V} / 200 \mathrm{M} \Omega$ & 0.16 & \\
\hline & $1000 \mathrm{~V} / 400 \mathrm{M} \Omega$ & 0.24 & \\
\hline \multirow[t]{3}{*}{ Kerosene } & $250 \mathrm{~V} / 100 \mathrm{M} \Omega$ & $\infty$ & \multirow{3}{*}{$\begin{array}{c}\text { Kerosene at ambient } \\
\text { temperature }\left(25^{\circ} \mathrm{C}\right)\end{array}$} \\
\hline & $500 \mathrm{~V} / 200 \mathrm{M} \Omega$ & $\infty$ & \\
\hline & $1000 \mathrm{~V} / 400 \mathrm{M} \Omega$ & $\infty$ & \\
\hline
\end{tabular}

\subsection{Speed and Current Characteristics}

The first characteristic to be investigated was the relationship between rotor speed and motor supply current. The LabVIEW software was used to record voltage signals representing speed and current at a scan period of 0.05 seconds and 400 samples per channel. During the experiment the motor supply current was increased gradually as shown in Figure 7 to provide a range of speed-current points.

The original instantaneous current data contained a significant amount of noise introduced by the current transducer. This noise was filtered using a moving average filter on the spreadsheet data. However the resulting current signal is certainly not a smooth curve with constant gradient. Adjusting the motor supply current manually accounts for the small amplitude variations in the waveform but the larger deviations are believed to be an inherent effect of the motor operating requirements. For motoring operation to occur there must be a continuous path for electrical current into one bearing, through the rotor and out of the other bearing. However whilst rotating, point contacts and current paths can be established and broken at a rapid rate introducing discontinuities in current flow and therefore instability in the current waveform. 


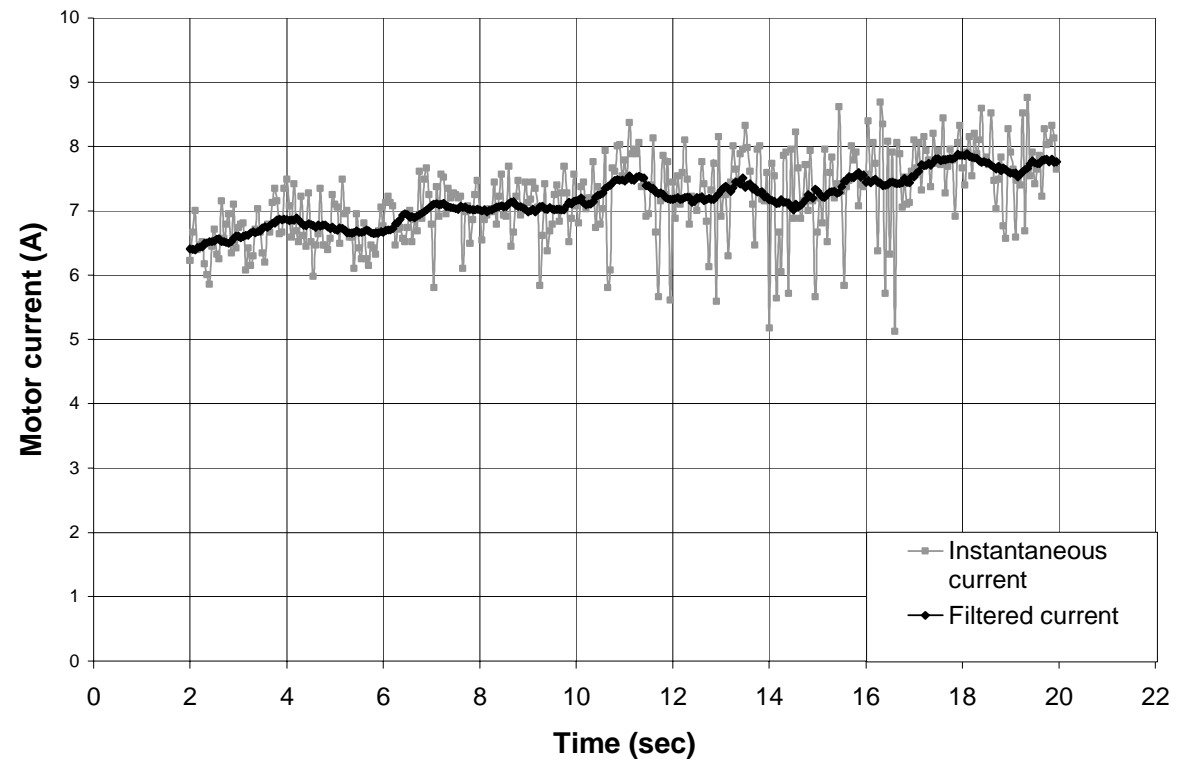

Figure 7. Instantaneous and filtered motor supply current waveforms. Direct current was increased gradually by hand from $6.5 \mathrm{~A}$ to 7.8A to observed the effect on rotor speed.

Figure 8 shows the resultant speed versus time characteristic. The plots identify a direct correlation between speed and current that appear to be roughly linear, unfortunately their inaccuracy prevents the conclusion of a mathematical relationship between the two quantities.

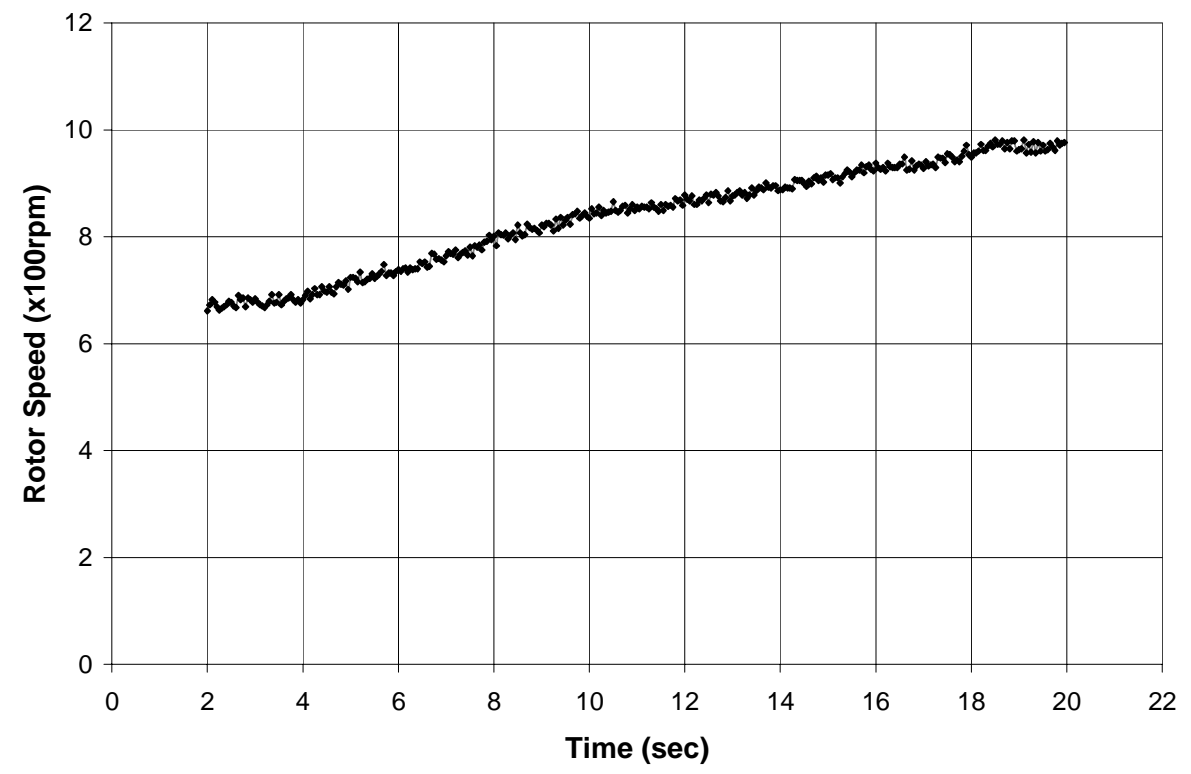

Figure 8. Rotor speed waveform for increasing supply current from 6.5A to 7.7A dc. Speed measurement performed over a 20 second interval on the second ball-bearing motor operating in kerosene. Maximum speed reached was approximately $1000 \mathrm{rpm}$. 


\subsection{Torque and Speed Characteristics}

The friction and windage torque against angular velocity curve for the dc-machine and Huber motor is shown in Figure 9 indicating a relatively constant torque over the speed range $0-500 \mathrm{rad} / \mathrm{s}$. The ball-bearing motor must exceed this torque in order to rotate. This test was performed on a motor with new bearings submerged in kerosene to incorporate the torque required to overcome liquid drag. Therefore, ideally a second friction and windage torque curve would be obtained after the acceleration torque measurement (involving motor excitation) to identify possible effects of bearing degradation and observe the repeatability of the test - this work is presently ongoing.

The dynamic acceleration torque measurement was carried for two different values of constant motor supply current, 16.7 A-dc and $30.5 \mathrm{~A}$-dc. Transient angular velocity versus time signals were captured using the LabVIEW speed measurement virtual instrument and are both displayed in Figure 10. It is obvious from these curves that increasing the value of constant motor supply current reduces the time taken for the rotor to reach a steady state operating speed. It is also valuable to notice that the general shape of the transient response is maintained and the major difference being the transition time from minimum to maximum angular velocity. Note that the steady-state speed attained is slightly greater with the higher current and that the acceleration torques are similar at the low speeds but diverge at higher speeds.

The characteristics of Figure 10 were then differentiated to obtain angular acceleration versus time values, which were then multiplied by the motor moment of inertia to find acceleration torque. The resultant data was then plotted against angular velocity giving the two acceleration torque versus speed characteristics of Figure 11. Extensive filtering was required to remove much of the noise created during differentiation.

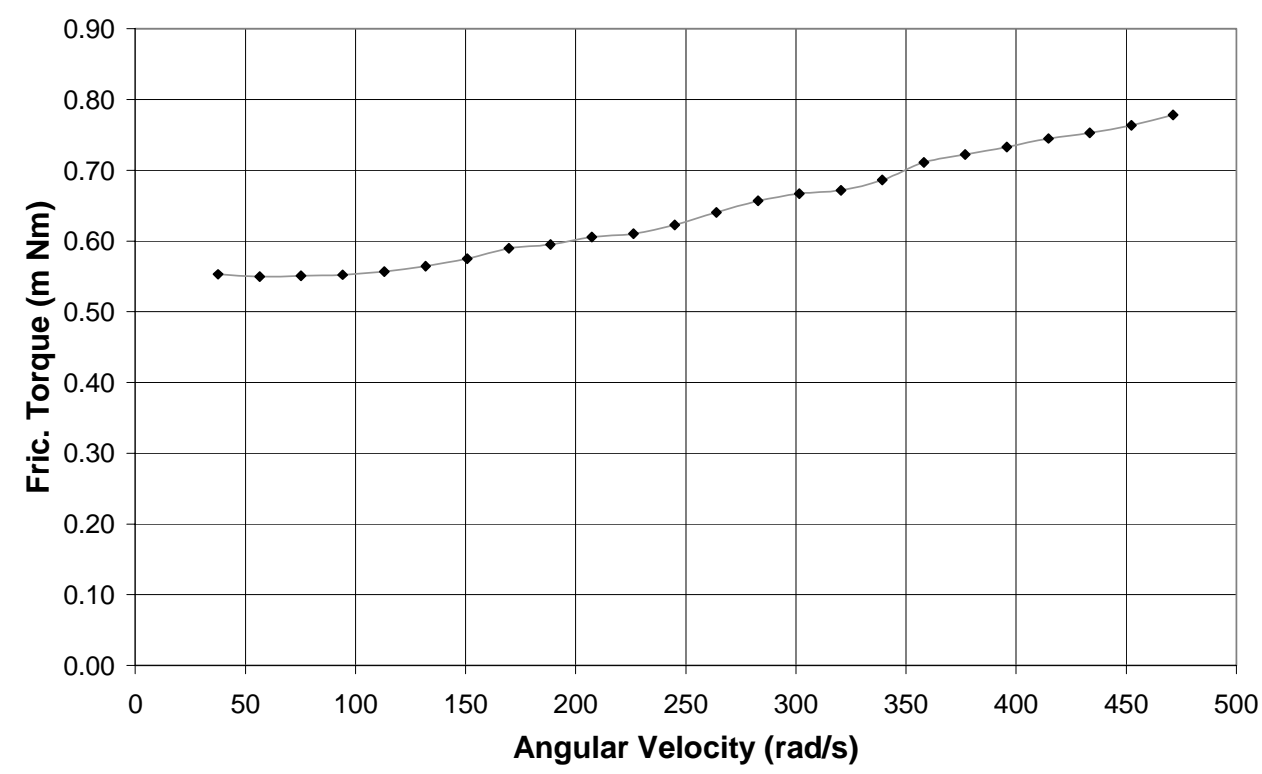

Figure 9. Friction and windage torque characteristic of second ball-bearing motor and dc-machine. DC-machine used to drive the unexcited Huber motor immersed in kerosene. Armature current of dc-machine was used to calculate system torque via equation (8) and back EMF constant $\mathrm{k}=6.9114 \times 10^{-3}$. 


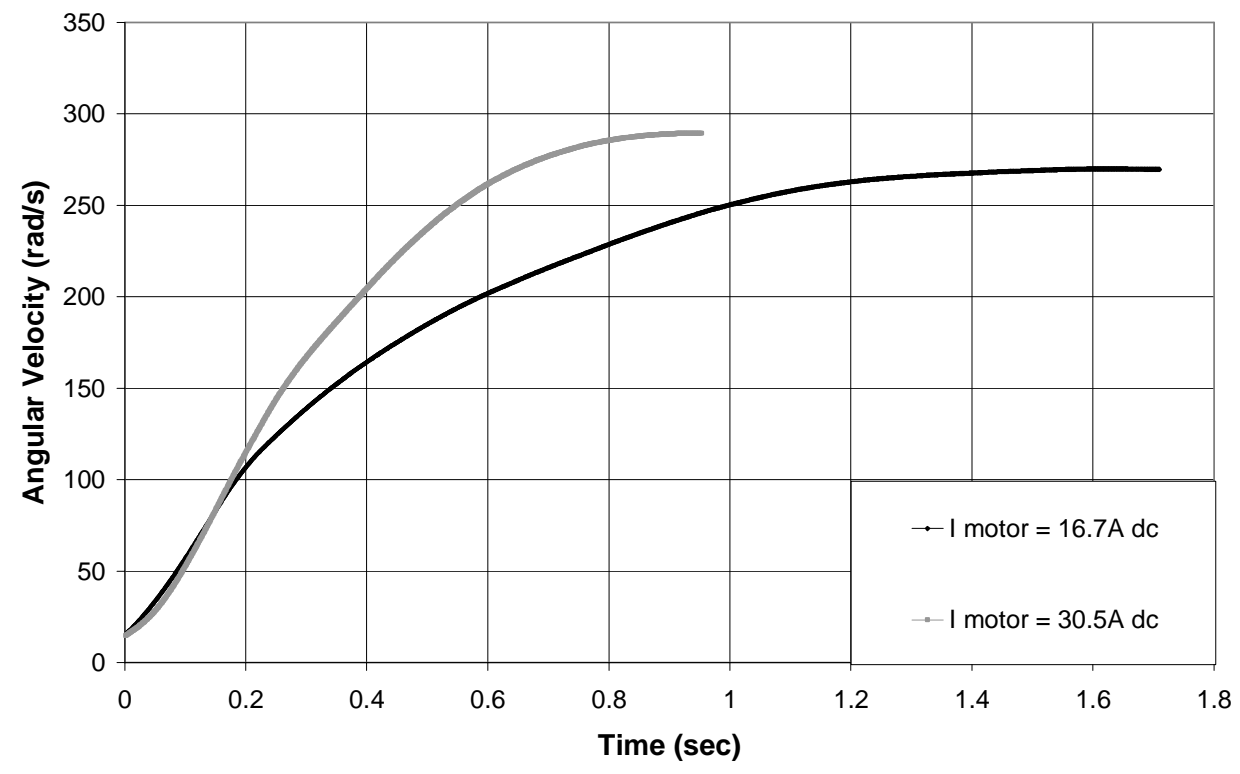

Figure 10. Transient angular velocity characteristics for second ball-bearing motor used to determine the acceleration torque produced. Motor operated at 16.7A and 30.5A dc in kerosene heat sink fluid. Test time duration long enough to allow a maximum steady state speed to be reached.

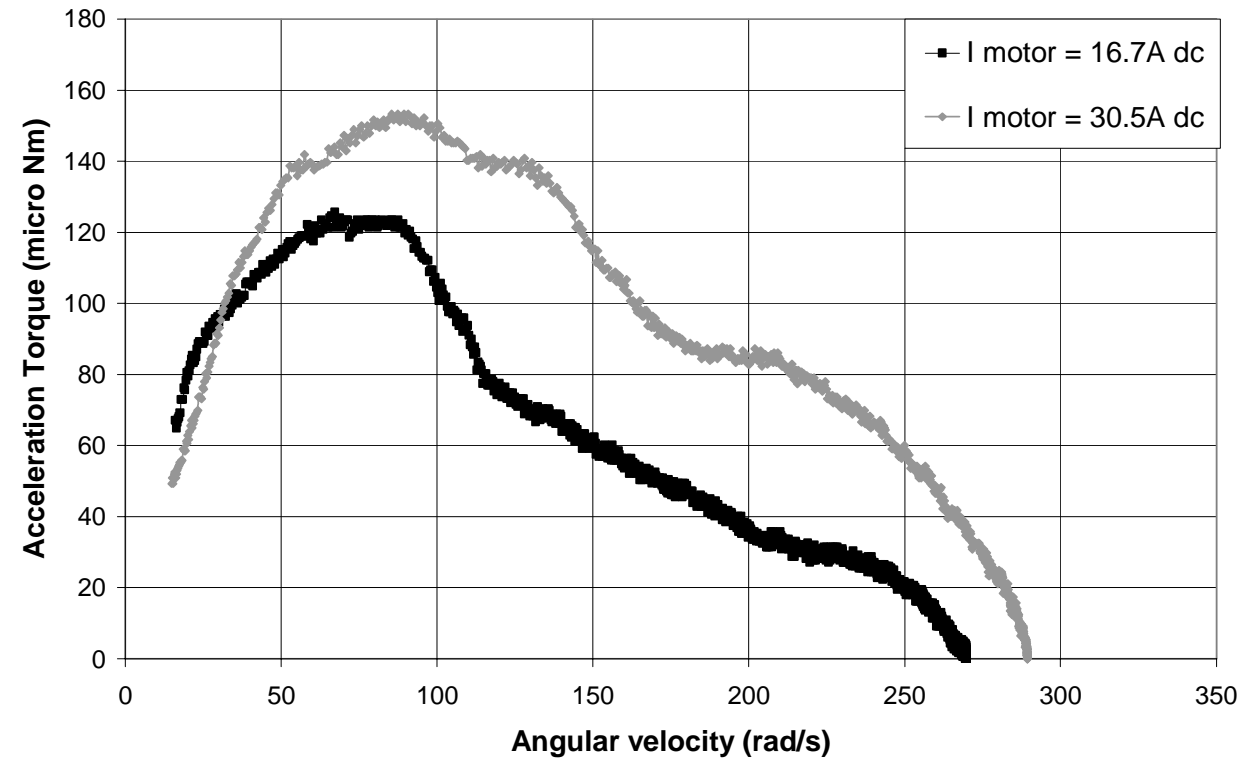

Figure 11. Acceleration torque versus angular velocity characteristic for the second ball-bearing motor design while immersed in kerosene. The test was performed for both 16.7A and 30.5A direct current on previously unused miniature bearings. 
Again, very similar shaped graphs were obtained for the two supply current settings, which suggests a constant increase in acceleration torque with increased motor current over the speed range. Further tests at different supply currents will allow firmer conclusions on the torque versus current characteristics to be determined.

The total dynamic torque versus speed characteristic for the motor can be calculated based on the results from Figures 9 and 11 .

\section{FUTURE WORK}

In this work, considerable time was taken to implement the fast data recording methods and to construct apparatus that will facilitate experimental data collection over the shortest possible time. In doing so, the accuracy and repeatability of the results is dramatically improved from those obtained in previous investigations giving a greater likelihood of identifying the physical nature behind the Huber effect.

The main problems encountered were shaft misalignment in the prototype ball-bearing motor design, high conductivity of the silicone oil, noise introduced into results from analysis and equipment and thermal expansion of the motor components limiting the number of consecutive tests that could be performed.

Future work should include a specific investigation into the spark discharge theory by operating the Huber motor immersed in fluid inside a vacuum. The rotor speed measurements should be recorded using the method described in this paper and the vacuum slowly released back to atmospheric pressure. By observing what happens to the rotor speed during this time, conclusions should be possible about the validity of the spark discharge theory. The effect of variable frequency alternating current on motor performance would also be useful as this involves changing electric and magnetic fields.

Ultimately, a motor design of extremely small dimension must be developed and tested to conclude whether micromotors operating under the Huber effect are a feasible means of performing mechanical motion.

\section{CONCLUSION}

A prototype ball-bearing motor design has been successfully developed with a maximum recorded speed of around $2800 \mathrm{rpm}$ at 30.5 A-dc. Initial torque and speed characteristics were obtained and describe the dynamic nature of the ball-bearing motors' operation and hence the physical interpretation of the Huber effect. The experimental results were also found to be reasonably repeatable, which can often be hard to achieve for electromechanical devices of small dimensions when the influences of temperature and high current can have huge impacts on material shape and friction properties. The Huber motor was found to produce torque in the order of hundreds of micro Newton meters. The benefit of an automated data acquisition process has also been demonstrated and this process will form the basis of future research into the Huber effect.

\section{ACKNOWLEDGMENTS}

The authors would like to thank the Electrical and Electronic Engineering Department technical staff for their fluent guidance and assistance regarding motor construction and experiment execution. In particular, thanks to Bill Finch, Ian Linke, Gordon Allison, Bernard Dumuid and Stephen Guest.

\section{REFERENCES}

1. Polivanov, K.M., Netushil, A.V. and Tatarinova, N.V., "Huber's electromechanical effect.” Elecktrichestvo, No. 8, pp.7276, 1973.

2. Milroy, R.A., "Hydrodynamic gyroscope," J. Appl. Mech., Vol. 34, p.525, 1967.

3. Gruenburg, H., "The ball bearing as a motor." Am. J. Phys., Vol. 46, pp.1213-1219, 1973.

4. Weenink, M.P.H., "The electromagnetic torque on axially symmetric rotating metal cylinders or spheres," Appl. Sci. Res., Vol. 37, pp.171-182, 1981.

5. Moyssides, F.G. and Hatzikonstantinou, P., "Study of electrical characteristics of the ball bearing motor." IEEE Trans. Magn., Vol. 26, No. 4, pp.1274-1281, 1990. 
6. Moyssides, F.G. and Hatzikonstantinou, P., "Ball bearing motors,” IEEE Trans. Magn., Vol. 33, No. 6, pp.4566-4569, 1997.

7. Watson, D.B., Williams, M.R. and Crimp, C.S., "Ball-bearing motors," IEE Proc.-A, Vol. 140, No. 4, pp.281-286, 1993.

8. Marinov, S., "The intriguing ball bearing motor," Electronics and Wireless World, pp. 356-357, April 1989.

9. Walker, M.B., Chambers Science and Technology Dictionary, 1994 reprint, (Chamber Cambridge 1988), p515.

10. NETUSHIL, A.V.: "The electromechanical effect of Huber and its development". Elec. Tech., No. 3, pp.57-64, 1992.

11. MILLS, A.A.: " The ball-bearing electric motor". Phys. Educ., Vol. 15, pp.102-103, 1980.

12. HATSIKONSTANTINOU, P. and MOYSSIDES, P.G.: "Explanation of the ball bearing motor and exact solutions of the related Maxwell equations". J. Phys. A: Math. Gen., Vol. 23, pp.3183-3197, 1990.

13. MOYSSIDES, P.G.: "Electrical characteristics of two discs operating as a motor". I.E.E.E Trans On Mag., Vol. 28, No. 3, pp.1870-1876, 1992.

14. WATSON, D.B. and WATSON, A.M.: "Linear ball-bearing motor". I.E.E. Proc.-Sci. Meas. Technol., Vol. 141, No. 3 , pp.224-228, 1994.

15. WATSON, D.B. and WATSON, A.M.: "Non-ferromagnetic linear ball-bearing motors". J. Phys. D: Appl. Phys., Vol. 29, pp.529-532, 1996.

16. WATSON, D.B.: "An explanation of the ball-bearing motor". Int. J. Electr. Eng. Educ., Vol. 28, pp.186-188, 1991.

17. WATSON, D.B.: "The force on an electrically conducting cylinder rolling on parallel rails". J. Phys. D: Appl. Phys., Vol. 30, pp.2176-2182, 1997.

18. RABINOVICI, R.I. and KAPLAN, B.Z.: "Effective magnetisation and forces due to eddy currents". I.E.E.E. Trans. On Mag., Vol. 28, No. 3, pp.1863-1869, 1992.

19. The ball-bearing electric motor, URL: www.netcomuk.co.uk/ wwl/bbmotor.html, last modified on: 11 Feb 2000.

20. Shen, Y., Tay B.K., Thompson B., Soong W.L., B.R. Davis and D. Abbott, "Investigation of the Huber effect and its application to micromotors," Proc. SPIE Electronics and Structures for MEMS, Vol. 3891, pp.178-183, Oct. 1999. 